Revista Destaques Acadêmicos, Lajeado, v. 9, n. 3, 2017. ISSN 2176-3070

DOI: http://dx.doi.org/10.22410/issn.2176-3070.v9i3a2017.1462

www.univates.br/revistas

\title{
PERFIL DA APTIDÃO FÍSICA, DO ESTADO NUTRICIONAL E DO NÍVEL DE ATIVIDADE FÍSICA DE CRIANÇAS DE ESCOLAS MUNICIPAIS DA CIDADE DE VENÂNCIO AIRES-RS
}

\author{
Ana Paula Bogorni ${ }^{1}$, Higor Rissini Ferrari ${ }^{2}$, Luiz Gabriel da Silva ${ }^{3}$, \\ Jéssica Luana Dornelles da Costa ${ }^{4}$, Caroline Pieta Dias ${ }^{5}$, \\ Carlos Leandro Tiggemann ${ }^{6}$
}

\begin{abstract}
Resumo: O estudo tem como objetivo identificar o perfil da aptidão física, do estado nutricional e do nível de atividade física de crianças de escolas municipais da cidade de Venâncio Aires-RS. Participaram do estudo 89 escolares da rede municipal da cidade de Venâncio Aires, tendo a faixa etária de 8 a 13 anos. Foram realizados os testes de aptidão física relacionados à saúde e o do estado nutricional, utilizando a bateria de teste do PROESP-Br e aplicado o questionário de atividade física. Os resultados encontrados nos indicam que 70,8\% dos escolares estão classificados na zona saudável na variável referente ao índice de massa corporal, $46,1 \%$ na flexibilidade, $40,4 \%$ na resistência abdominal, 56,2\% na resistência aeróbica e 44,2\% classificados como ativos. Baseado nos resultados de nosso estudo podemos concluir que a maioria dos escolares não apresentam níveis adequados de aptidão física relacionados à saúde e de atividade física.
\end{abstract}

Palavras-chave: Escolares. Aptidão física. Estado nutricional. Atividade física.

1 Profissional de Educação Física Bacharelado - Univates - Lajeado/RS. abogorni@universo.univates.br

2 Graduando do Curso de Educação Física Bacharelado - Univates - Lajeado/RS. higor.ferrari@univates.br

3 Graduando do Curso de Educação Física Bacharelado - Univates - Lajeado/RS. luiz.silva4@univates.br

4 Profissional de Educação Física Licenciatura - Univates - Lajeado/RS. jluana@univates.br

5 Doutora em Ciência do Movimento Humano (UFRGS); professora do Curso de Educação Física - ESEF/UFRGS- Porto Alegre/RS. carolpieta@yahoo.com.br

6 Doutor em Ciência do Movimento Humano (UFRGS); professor do Curso de Educação Física - Univates-Lajeado/RS e FSG-Caxias do Sul/RS. cltiggemann@univates.br 


\section{INTRODUÇÃO}

A aptidão física pode ser compreendida como a capacidade do indivíduo em realizar suas tarefas diárias com vigor, sem apresentar um cansaço excessivo, garantindo assim uma sobrevivência no meio ambiente em que vive (BOUCHARD, 1990). A aptidão física pode ser dividida em dois grupos, sendo um relacionado às habilidades motoras (performance) e o outro relacionado à saúde.

A aptidão física associada à performance é compreendida como a capacidade de um indivíduo em realizar atividades físicas que exijam um desempenho muscular demonstrando uma aptidão individual, onde suas variáveis estão voltadas para as habilidades desportivas tais como a agilidade, equilíbrio, coordenação motora, potência e velocidade (BOUCHARD, 1990). Em contrapartida, a aptidão física relacionada à saúde é definida como a capacidade do nosso organismo (coração, pulmão, vasos sanguíneos e músculos) a resistirem às doenças degenerativas e conseguirem realizar atividades cotidianas, constituída pelas variáveis resistência cardiorrespiratória, força, resistência muscular e a flexibilidade (ACSM, 1996).

Manter um adequado nível de aptidão física traz vários benefícios para os indivíduos, entre eles pode-se destacar uma menor incidência de doenças crônicas degenerativas, como elevados níveis de colesterol sanguíneo, hipertensão, acidente vascular cerebral, doenças coronarianas e obesidade (NAHAS, 2001). Desta forma, é importante que a prevenção dessas doenças seja iniciada na infância e adolescência através da prática de atividades físicas regulares, pois os hábitos que são estimulados nessa fase geralmente são mantidos durante a vida adulta (AAHPERD, 1988).

Apesar dos inúmeros benefícios que a atividade física traz na vida dos indivíduos, o sedentarismo está cada vez mais presente na vida das pessoas, sendo que o aumento da urbanização na sociedade influenciou na diminuição dos níveis de aptidão física, diminuindo os espaços para a prática do esporte e de atividades física (PETROSKI et al., 2012). Além disso, as mudanças do cotidiano como as facilidades dos eletrônicos no trabalho doméstico, transporte automotor, computadorização do trabalho industrial e as ocupações no momento de lazer com atividades passivas como assistir televisão e usar o computador também contribuíram para o aumento do sedentarismo (WERK et al., 2009).

Outro fator que vem preocupando a saúde de crianças e adolescentes refere-se ao estado nutricional, pois, a obesidade é caracterizada pelo excessivo acúmulo de gordura ocasionada em grande parte por um desequilíbrio crônico (DANIELS et al., 2005; LIMA, GLANER, 2006). Por isso, manter os níveis de composição corporal é de extrema importância, pois, os indivíduos obesos podem apresentar várias doenças, dentre elas a elevação do nível de colesterol sanguíneo, vários tipos de câncer, além de problemas psicológicos e sociais 
(LIMA, GLANER, 2006; BOUCHARD et al., 1991). Considerando os diversos aspectos supracitados, a prevenção destas doenças já deve ser iniciada desde a infância e juventude, pois, acredita-se que doença degenerativa já tem seu período de incubação nessa fase e algumas delas já podem se manifestar na infância, como a obesidade e a hipertensão (AAHPERD, 1988).

Em estudo realizado com 7.507 escolares de 7 a 10 anos de idade, de cinco regiões brasileiras (Norte, Nordeste, Centro-Oeste, Sudeste e Sul), mostrou que houve uma elevada prevalência de escolares que não atendem aos critérios de saúde da classificação geral da proposta adotada nos testes de flexibilidade, força/resistência muscular e aptidão cardiorrespiratória (PELEGRINI et al., 2011). O resultado indicou que aproximadamente $96 \%$ dos escolares não atingiram os patamares desejados de saúde. Em relação ao nível de atividade física, o estudo de Da Silveira Bueno et al. (2015) analisou o nível de atividade física de 147 escolares de 6 a 10 anos de idade, das cidades de Encantado/RS e Vespasiano Correa/RS, e constatou que $30,6 \%$ das crianças estão classificadas como ativas e $69,4 \%$ classificadas como inativas.

Considerando a importância de manter os níveis de aptidão física, o estado nutricional e nível de atividade física adequados, tendo em mente seus benefícios, um dos melhores estímulos está ligado à avaliação dos mesmos, fornecendo informações dos indivíduos sobre sua condição física, identificando as que possuem algum tipo de risco potencial já na infância. Sendo assim, o estudo tem como objetivo identificar o perfil da aptidão física, do estado nutricional e do nível de atividade física de crianças de escolas municipais da cidade de Venâncio Aires-RS.

\section{PROCEDIMENTOS METODOLÓGICOS}

Esse estudo foi desenvolvido por meio de uma abordagem de pesquisa do tipo descritiva quantitativa de corte transversal. Participaram do estudo 89 escolares da rede municipal da cidade de Venâncio Aires, destes, 42,7\% residem em área rural e 57,3 em área urbana, tendo a faixa etária de 8 a 13 anos, sendo $44,9 \%$ do sexo feminino e $55,1 \%$ do sexo masculino. Para a avaliação dos escolares foram escolhidas por acessibilidade quatro escolas municipais da cidade de Venâncio Aires, sendo, duas localizadas na área urbana e duas na área rural. Como critério de inclusão para participar do estudo, o aluno deveria estar matriculado e frequentando a escola no ano letivo de 2016, apresentar o termo de consentimento assinado pelos pais ou responsáveis, não apresentar nenhum problema de saúde que o impedisse de realizar os testes. O presente estudo teve a autorização da Secretaria da Educação do Município de Venâncio Aires e a aprovação do Comitê de Ética em Pesquisa (Coep) da Univates (parecer $\mathrm{n}^{\circ}$ 1.339.552).

Para a realização dos testes foram realizadas três visitas em cada escola, onde primeiramente foi explicado a proposta do estudo e os testes que seriam 
aplicados, após foi feito o convite aos escolares para a participação no estudo. Os interessados em participar levaram o Termo de Consentimento Livre e Esclarecido para os pais ou responsáveis assinarem permitindo a participação no estudo. Na segunda visita, foi aplicado o questionário de atividade física aos escolares, os testes de aptidão física (flexibilidade e resistência muscular localizada) e do estado nutricional (massa corporal e estatura), sendo todos realizados individualmente em uma sala de aula para evitar possíveis constrangimentos aos escolares. Na última visita realizou-se o último teste, de capacidade aeróbica, sendo aplicado em duplas na quadra de esportes das escolas. Todos os testes foram aplicados no turno de aula dos escolares, nos períodos das aulas de educação física e por períodos disponibilizados pela professora titular, sempre com sua presença.

Para a avaliação da atividade física dos estudantes foi aplicado o questionário de atividade física PAQ-C (CROCKER et al., 1997), que tem como objetivo investigar o nível de atividade física dos escolares, ao longo dos setes dias que antecedem a aplicação do questionário incluindo o final de semana. $\mathrm{O}$ questionário é composto por nove questões sobre a prática de esportes, jogos, atividades físicas na escola e no tempo de lazer, cada questão tem valor de 1 a 5 e o escore final é obtido pela média das questões, sendo representada por muito sedentário 1 e muito ativo 5, os escores 2,3,4 indicam as categorias sedentários, moderadamente ativo e ativos. Dessa forma os estudantes foram classificados como ativos com escore $\geq 3$, ou sedentários com escore $<3$.

O estado nutricional foi avaliado através do índice de massa corporal (IMC) usando o cálculo da massa corporal dividida pela estatura ao quadrado, sendo os resultados classificados como "zona de risco á saúde" e "zona saudável", conforme a tabela normativa apresentada para cada idade e sexo, disponível no Manual do PROESP-Br (2007). Para avaliar a massa corporal utilizou-se uma balança portátil (marca WISO e modelo W721), sendo solicitado aos escolares que ficassem posicionados de frente para a balança, pés juntos e braços estendidos ao longo do corpo, os estudantes ficaram descalços e vestindo roupas leves. A avaliação da estatura foi por meio de um estadiômetro (marca Belmak e modelo Est220), onde os estudantes ficaram descalços em posição ereta, braços ao longo do corpo.

Já para a avaliação do nível de aptidão física utilizou-se a bateria de teste do PROESP-Br (2007), no qual os testes aplicados foram de aptidão física relacionado à saúde sendo o de flexibilidade, resistência muscular localizada e capacidade aeróbica. A flexibilidade foi avaliada através do teste de sentare-alcançar, onde foi estendida e fixada uma fita métrica no solo, os escolares sentavam-se no solo com os joelhos estendidos e as mãos sobrepostas, inclinando-se lentamente para frente o mais distante possível, foram realizadas duas tentativas, utilizando o melhor resultado.

O teste de resistência muscular localizada foi avaliado através do teste de abdominal em um minuto, o estudante deitou-se sobre um colchonete em 
decúbito dorsal com os joelhos flexionados a 45 graus e com os braços cruzados sobre o tórax, o estudante realizava o movimento de flexão do tronco até tocar os cotovelos na coxa retornando à posição inicial. O resultado foi contabilizado pelo número de repetições completas realizados em um minuto. Para a avaliação da capacidade aeróbica utilizou-se o teste de corrida de seis minutos, o teste foi realizado mediante corrida de seis minutos, o estudante correu a maior distância possível nesse período de tempo, sendo permitido intercalar com caminhada conforme necessidade do estudante. Ao final foi registrada a distância percorrida.

Como critério de classificação do nível de aptidão física dos participantes, os resultados foram classificados como "zona de risco à saúde" e "zona saudável", conforme a tabela normativa apresentada para cada idade e sexo, disponível no Manual do PROESP-Br (2007). A análise dos dados foi realizada por meio de estatística descritiva, com valores mínimos, máximos, média, desvio padrão e distribuição de frequência.

\section{RESULTADOS E DISCUSSÕES}

O estudo teve por objetivo identificar o perfil da aptidão física, do estado nutricional e do nível de atividade física de crianças de escolas municipais da cidade de Venâncio Aires/RS. Os resultados encontrados estão apresentados na tabela 1, nos indicando que $70,8 \%$ dos escolares estão classificados na zona saudável na variável referente ao IMC, $46,1 \%$ na flexibilidade, $40,4 \%$ na resistência abdominal, 56,2\% na resistência aeróbica e 44,2\% classificados como ativos.

A obesidade entre crianças e adolescentes têm apresentado crescente prevalência nas últimas décadas e já é considerada um dos maiores problemas da saúde pública em âmbito mundial, podendo trazer repercussões na vida dos mesmos, tais como os transtornos sociais e doenças coronarianas como a hipertensão e diabetes (VASQUES, MARTINS, AZEVEDO, 2004). Apesar dos dados apresentarem que a obesidade vem aumentando ao longo dos anos, os resultados encontrados no nosso estudo mostraram que 70,8\% dos escolares encontram-se na zona saudável e $29,2 \%$ na zona de risco à saúde. Já o estudo realizado com 69 escolares de 7 a 11 anos de Brasília-DF, apresentou que 82,6\% dos escolares encontram-se na zona saudável, enquanto 17,4\% encontram-se na zona de risco à saúde (PEREIRA et al., 2012). Em outro estudo, com 147 crianças de 6 a 10 anos, das cidades de Encantado-RS e Vespasiano CorrêaRS classificou o índice de massa corporal (IMC) como normal, sobrepeso e obesidade, e constatou que 53,7\% dos escolares estão na classificação normal, $20,4 \%$ estão com sobrepeso e $25,9 \%$ estão considerados obesos (DA SILVEIRA BUENO et al., 2015). 
Tabela 01. Tabela descritiva com valores mínimos e máximos, média e desvio padrão e distribuição percentual dos dados gerais

\begin{tabular}{l|c|c|c}
\hline \multicolumn{1}{c|}{ Variável } & Mínimo & Máximo & Média \pm DP \\
\hline Idade (anos) & 8 & 13 & $9,80 \pm 0,97$ \\
\hline Massa Corporal (kg) & 23,1 & 75,5 & $37,80 \pm 10,23$ \\
\hline Estatura (m) & 1,25 & 1,65 & $1,42 \pm 0,07$ \\
\hline IMC (kg/m ${ }^{\text {) }}$ & 11,75 & 29,57 & $18,67 \pm 3,86$ \\
\hline Risco à saúde & - & - & $29,2 \%$ \\
\hline Zona Saudável & - & - & $70,8 \%$ \\
\hline Flexibilidade (cm) & 11 & 50 & $30,18 \pm 7,78$ \\
\hline Risco à saúde & - & - & $53,9 \%$ \\
\hline Zona saudável & - & - & $46,1 \%$ \\
\hline Resistência abdominal (rep) & 08 & 38 & $24,38 \pm 6,40$ \\
\hline Risco à saúde & - & - & $59,6 \%$ \\
\hline Zona saudável & - & - & $40,4 \%$ \\
\hline Resistência aeróbica (m) & 510 & 1539 & $851,71 \pm 221,21$ \\
\hline Risco à saúde & - & - & $43,8 \%$ \\
\hline Zona saudável & - & - & $56,2 \%$ \\
\hline Nível de atividade física (u.a) & 1,48 & 4,30 & $2,80 \pm 0,64$ \\
\hline Sedentários & - & - & $55,1 \%$ \\
\hline Ativos & - & - & $44,9 \%$ \\
\hline
\end{tabular}

Legenda: IMC = índice de massa corporal; u.a. = unidade arbitrária.

De acordo com as variáveis da aptidão física relacionada à saúde, a flexibilidade é um importante componente, expressando a capacidade da elasticidade demonstrada pelos músculos e tecidos conectivos ligados a mobilidade articular (DE ARAÚJO, 2008). Manter adequados níveis de flexibilidade está associado à prevenção de doenças posturais e menor incidência de lesões, além de ajudar na realização das tarefas diárias como pegar algum objeto, amarrar os calçados, entre outros (WERK et al., 2009).

No presente estudo foi constatado que $53,9 \%$ dos escolares encontramse na zona de risco a saúde e $46,1 \%$ na zona saudável. No estudo de Pereira et al. (2012) foi constatado que $47,8 \%$ dos escolares não atenderam aos critérios de saúde e 52,2\% atenderam os critérios de saúde. A flexibilidade diminui até a puberdade e tende a aumentar posteriormente, voltando a diminuir na idade adulta (DE ARAÚJO, 2008). Estudos têm demonstrado que a diferença na flexibilidade pode ser caracterizada como multifatorial, levando em consideração as medidas antropométricas, a composição corporal, a genética, 
a cultura e uma possível patologia de cada indivíduo (GRANT et al., 2001; ALARANTA et al., 1994; GRAHAME, 2001; PEREIRA et al., 2012).

Quando nos referimos à variável resistência e a força muscular localizada na região abdominal, as mesmas são tão importantes quanto os demais componentes de aptidão física, pois para realizar tarefas do cotidiano há uma necessidade mínima de capacidade muscular (GRANT et al., 2001). Manter adequados níveis de resistência abdominal evitará futuros problemas posturais aos indivíduos (GRANT et al., 2001). Em nosso estudo, quando avaliado esse componente, 59,6\% dos escolares foram classificados com risco a saúde e $40,4 \%$ classificados na zona saudável.

No estudo de Pelegrini et al. (2011) foi observado que mais de 50\% dos escolares também encontravam-se classificados abaixo dos critérios de saúde em relação a classificação geral. Porém em outro estudo com escolares, encontrouse um percentual maior de escolares na zona saudável, $65,2 \%$ dos escolares atendem os critérios de saúde e $34,8 \%$ não atendem os critérios de saúde (PEREIRA et al., 2012). Esse fato pode ser explicado pela hipótese dos hábitos de atividades serem diferentes em cada região do país. Em estudo realizado em Moçambique com crianças que residem em diferentes regiões socioeconômicas indicou menor desempenho no teste de resistência e força abdominal em crianças pertencentes a regiões menos favorecidas economicamente (PRISTA, MARQUES, MAIA, 1997). Essa hipótese merece ser mais investigada, por vez que não foi controlada no presente estudo.

A variável resistência aeróbia está relacionada ao funcionamento do coração, pulmão e sistema vascular, o que requer o uso considerável do sistema circulatório e respiratório (GALLAHUE, OZMUN, GOODWAY, 2013). Em nosso estudo esta variável indicou que $43,8 \%$ dos escolares estão classificados na zona de risco a saúde e $56,2 \%$ na zona saudável, o que é muito positivo, pois seus níveis adequados contribuem para o não aparecimento de possíveis doenças crônicas degenerativas. Em estudo de Pelerini et al. (2011) realizado com escolares de cinco regiões brasileiras, demonstrou que aproximadamente $80 \%$ dos escolares não atingiram os critérios estabelecidos para a saúde em relação à aptidão cardiorrespiratória. Para o autor esse é um dado considerado preocupante, pois uma baixa aptidão cardiorrespiratória está associada a elevados níveis de colesterol e triglicerídeos, aumento da pressão arterial e maior risco de obesidade.

Quando nos referimos à prática de atividade física, a mesma deveria ser indispensável para qualquer indivíduo, principalmente para as crianças e adolescentes, pois é nessa fase que a prática de atividade física pode atuar contra o surgimento de doenças como a obesidade, diabetes e hipertensão na vida adulta (ALARANTA et al., 1994). Contudo não foi o que encontramos em nosso estudo, no qual $55,1 \%$ dos estudantes foram classificados como sedentários e 44,9\% como ativos. Em estudo realizado em município próximo ao de Venâncio Aires, o percentual de sedentários também foi maior que os 
ativos, sendo $69,4 \%$ classificados como sedentários e 30,6\% considerados ativos (DA SILVEIRA BUENO et al., 2015). Em outro estudo com 325 alunos, sendo 123 meninos e 204 meninas de 14 a 15 anos de dezesseis escolares de Niterói/ RJ, aproximadamente $85 \%$ dos escolares do sexo masculino e $94 \%$ do sexo feminino foram classificados como sedentários (DA SILVA, MALINA, 2000).

O problema do baixo nível de atividade física tende a atingir o mundo inteiro, autores ressaltam a necessidade de aumentar os níveis de atividade física entre os escolares revendo seus hábitos sedentários, tais como, as horas excessivas em frente ao computador e assistindo televisão (DA SILVA, MALINA, 2000; TEIXEIRA et al., 2008).

\section{CONCLUSÃO}

De acordo com os resultados do nosso estudo, podemos concluir que a maioria dos escolares apresentam níveis inadequados de aptidão e de atividade física, contudo, o resultado do estado nutricional apresentou patamares satisfatórios estabelecidos para a saúde. Com isso percebemos que é de extrema importância a necessidade de enfatizar as políticas educacionais referentes à aptidão e atividade físicas nas escolas, com professores desenvolvendo atividades que estimulam as mesmas durante as aulas de educação física. Espera-se que os resultados encontrados neste estudo possam auxiliar em demais investigações sobre o estado nutricional, o nível de aptidão física e de atividade física, levando em conta a escassez de estudos relacionados ao assunto no Vale do Rio Pardo e Taquari.

\section{REFERÊNCIAS}

ALARANTA, H. et al. Flexibility of the spine: normative values of goniometric and tape measurements. Scandinavian journal of rehabilitation medicine, v. 26, n. 3, p. 147-154, 1994.

AMERICAN COLLEGE OF SPORTS MEDICINE et al. Manual para teste de esforço e prescrição de exercício físico. Rio de Janeiro: Revinter, 1996.

BOUCHARD, C. et al. genes in the constellation of determinants of regional fat distribution. International journal of obesity, 1991.

BOUCHARD, C. et al. Exercise, fitness, and health: a consensus of current knowledge: proceedings of the International Conference on Exercise, fitness, and health, May 29-June 3, 1988, Toronto, Canada. In: Exercise, fitness, and health: a consensus of current knowledge: proceedings of the International Conference on Exercise, fitness, and health, May 29-June 3, 1988, Toronto, Canada. Human Kinetics Publishers, 1990.

BRASIL, Projeto Esporte. Manual de aplicações de medidas e teste, normas e critérios de avaliação. 2007. 
CROCKER, P. R. et al. Measuring general levels of physical activity: preliminary evidence for the Physical Activity Questionnaire for Older Children. Medicine and science in sports and exercise, v. 29, n. 10, p. 1344-1349, 1997.

DA SILVA, R. C. R.; MALINA, Robert M. Nível de atividade física em adolescentes do Município de Niterói, Rio de Janeiro, Brasil Level of physical activity in adolescents from Niterói, Rio de Janeiro, Brazil. Cadernos de Saúde Pública, v. 16, n. 4, p. 10911097, 2000.

DA SILVEIRA BUENO, A. et al. AVALIAÇÃO DO PERFIL NUTRICIONAL E PRÁTICA DE ATIVIDADE FÍSICA DE ALUNOS DE SÉRIES INICIAIS DO ENSINO PÚBLICO E PRIVADO DOS MUNICÍPIOS DE ENCANTADO E VESPASIANO CORRÊA/RS. Revista Destaques Acadêmicos, v. 7, n. 3, 2015.

DANIELS, S. R. et al. Overweight in children and adolescents. Circulation, v. 111, n. 15, p. 1999-2012, 2005.

DE ARAÚJO, C. G. S. Avaliação da flexibilidade: valores normativos do flexiteste dos 5 aos 91 anos de idade. Arquivos Brasileiros de Cardiologia, Rio de Janeiro, v. 90, n. 4, p. 280-287, 2008.

GALLAHUE, D. L.; OZMUN, J. C.; GOODWAY, Jackie D. Compreendendo o desenvolvimento motor-: bebês, crianças, adolescentes e adultos. AMGH Editora, 2013.

GRAHAME, R. Time to take hypermobility seriously (in adults and children). Rheumatology, v. 40, n. 5, p. 485-487, 2001.

GRANT, S. et al. A comparison of the anthropometric, strength, endurance and flexibility characteristics of female elite and recreational climbers and non-climbers. Journal of sports sciences, v. 19, n. 7, p. 499-505, 2001.

LIMA, W. A.; GLANER, M. F. PRINCIPAIS FATORES DE RISCO RELACIONADOS ÀS DOENÇAS CARDIOVASCULARES MAIN FACTORS OF RISK RELATED TO THE CARDIOVASCULAR DISEASES. Revista Brasileira de Cineantropometria e Desempenho Humano, v. 8, n. 1, p. 96-104, 2006.

NAHAS, M. V. Educação para um estilo de vida ativo nos programas de educação física. Atividade física, saúde e qualidade de vida. Londrina: Ed. Midiograf, 2001.

PEREIRA, C. H. et al. Aptidão física em escolares de uma unidade de ensino da rede pública de Brasília-DF. Revista Brasileira de Atividade Física \& Saúde, v. 16, n. 3, p. 223-227, 2012.

PETROSKI, E. L. et al. Associação entre baixos níveis de aptidão física e fatores sociodemográficos em adolescentes de área urbanas e rurais. Motricidade, v. 8, n. 1, 2012. 
PRISTA, A.; MARQUES, A. T.; MAIA, J. A. R. Relationship between physical activity, socioeconomic status, and physical fitness of 8-15-year-old youth from Mozambique. American Journal of Human Biology, v. 9, n. 4, p. 449-457, 1997.

TEIXEIRA, C. G. O. et al. Nível de atividade física nos períodos de aula e de férias em escolares de Anápolis-GO. Revista Brasileira de Ciência e Movimento, v. 13, n. 1, p. 45-50, 2008.

VASQUES, F.; MARTINS, F. C.; AZEVEDO, A. P. de. Aspectos psiquiátricos do tratamento da obesidade. Revista de Psiquiatria Clínica, v. 31, n. 4, p. 195-8, 2004.

WERK, R. et al. Aptidão física relacionada à saúde de crianças de uma escola estadual de Campo Grande/MS. Ciência, cuidado e saúde, v. 8, n. 1, p. 42-47, 2009. 